\title{
The church bridge project: Lessons learned from comparing the outcomes of an adapted church-based weight management intervention targeting african american adults
}

\author{
Jennifer L. Lemacks* ${ }^{* 1}$, Laurie L. Abbott ${ }^{2}$, Ashley N. Bryant ${ }^{1}$, Hwanseok Choi ${ }^{3}$, Penny A. Ralston ${ }^{4}$ \\ ${ }^{1}$ School of Kinesiology and Nutrition, The University of Southern Mississippi, Hattiesburg, Mississippi, United States \\ ${ }^{2}$ College of Nursing, The Florida State University, Tallahassee, Florida, United States \\ ${ }^{3}$ School of Health Professions, The University of Southern Mississippi, Hattiesburg, Mississippi, United States \\ ${ }^{4}$ Center on Better Health and Life for Underserved Populations, The Florida State University, Tallahassee, Florida, United States
}

Received: October 25, 2019

Accepted: February 2, 2020

Online Published: March 28, 2020

DOI: $10.5430 /$ jer.v5n2p37

URL: https://doi.org/10.5430/jer.v5n2p37

\begin{abstract}
Introduction: Church-based interventions improve weight-related outcomes among underserved populations. African Americans, particularly young to middle age adults, are seldom represented in the literature and are key health intervention targets to prevent obesity-related chronic disease. Thus, the objective of this study was to describe the lessons learned from comparing the intervention outcomes of a two-phase, weight management intervention targeting younger to middle aged adult African Americans in Mississippi.

Methods: A weight management intervention was tested and data were collected over two phases using the Bridge2U platform. Descriptive methods examined retention and attendance rates, and anthropometric and demographic data; Wilcoxon signed-rank and Fisher's exact tests examined group differences in pre and post outcomes.

Results: Attendance rates were 39.9 and $55.4 \%$ for phases 1 and 2, respectively. Differences were noted between those who dropped out of the phase 1 intervention and those who remained. Weight loss was significant at 5.6 and $4.8 \%$ of baseline body weight for phases 1 and 2, respectively. Significant changes were also noted among other variables.

Conclusions: Disease diagnosis, more intensive intervention format and technology-assisted delivery may be key factors for improved participation and engagement of young to middle aged African Americans in weight management interventions. This study provides future implications for weight management intervention and chronic disease prevention among young to middle aged adult African Americans in the Deep South.
\end{abstract}

Key Words: Obesity, Minority health, Nutrition, Physical activity, Health disparities

\section{INTRODUCTION}

Obesity is a major public health problem and a contributory risk factor for the major causes of death in the United States, including cardiovascular disease, diabetes, and cancer. ${ }^{[1,2]}$
The prevalence of obesity has substantially increased among American adults (from $30.5 \%$ in 2000 to $37.7 \%$ in 2014), and this trend is mirrored in the considerable percentage of children and adolescents, aged 2-19 years, considered over-

\footnotetext{
*Correspondence: Jennifer L. Lemacks; Email: Jennifer.Lemacks@usm.edu; Address: School of Kinesiology and Nutrition, The University of Southern Mississippi, Hattiesburg, Mississippi, United States
} 
weight $(16.2 \%)$ and obese $(17.2 \%) .{ }^{[1]}$ National health objectives aim toward reducing obesity rates, increasing the proportion of children and adults within a healthy weight range, and advancing health equity. ${ }^{[3]}$ The geographic clustering of acute and chronic disease risk factors are influenced by place of residence. ${ }^{[4,5]}$ For example, people living in the southern United States have greater obesity risk, and subsequently, the rates of obesity reported for the South $(31.2 \%)$ are the highest in the nation compared with regions in the Midwest $(30.7 \%)$, Northeast $(26.4 \%)$, and West $(25.2 \%) .^{[1,2,6]}$

There is a higher percentage of obese residents in rural areas $(35.6 \%)$ of the United States than urban locations $(30.4 \%){ }^{[6]}$ Located in the Deep South, the state of Mississippi has the highest obesity percentage (37\%), compared with other states and a notable obesity disparity among African Americans (44\%) compared with Caucasians (33\%). ${ }^{[2]}$ African Americans living in the rural South have disproportionately higher obesity rates and fewer support resources that facilitate lifestyle and body weight changes. ${ }^{[7]}$ Church-based interventions may increase social support and can concurrently be effective for improving diet, physical activity and weight outcomes. ${ }^{[8]}$ Despite the high prevalence of obesity in Mississippi, few articles discuss the effectiveness of faith-based obesity management interventions, especially among younger participants. ${ }^{[9-12]}$ The goal of this report is to bridge this gap by reporting the findings of an ongoing church-based obesity management study conducted among African American participants younger than 50 years of age. Two weight management interventions were implemented in south Mississippi with the second intervention (phase 2) being adapted based on the outcomes observed from the first intervention (phase 1). Thus, the objective of this study was to describe the lessons learned from comparing the intervention outcomes of a two-phase, weight management intervention targeting younger to middle aged adult African Americans in Mississippi.

\section{MethodS}

\subsection{Study overview}

This study builds on work previously described ${ }^{[13,14]}$ to include the theoretical framework and recruitment methods and baseline results for the Phase 1 intervention. The original primary aim of the Church Bridge Project was to assess the feasibility of a theory-driven (Socio-ecological, Transtheoretical and Health Belief Models), church-based intervention with a telehealth component to reduce obesity and related chronic disease disparities in rural, African Americans using the RE-AIM framework (Reach, Effectiveness, Adoption, Implementation, and Maintenance). The study originally recruited young to middle aged, African American adults (between 18 and 50 years of age) with children between 5 and 11 years of age to participate in a 12-session weight management intervention. The original recruitment goal for the study was a conservative 30 participants to account for a potential $30 \%$ attrition that would allow for retention of at least 20 participants in the study for pre and post measure assessment. All study protocol and procedures were approved by the Institutional Review Board. The National Institutes of Health definition considers this project a clinical trial which is registered at ClinicalTrials.gov (identifier: NCT02773069)

\subsection{Phase 1 intervention}

The development of the Church Bridge Project involved a community-academic partnership using a community-based participatory approach. ${ }^{[13]}$ The resulting Phase 1 intervention is described previously, ${ }^{[13]}$ including theoretical framework, recruitment methods, and baseline results. The 12 -session weight management intervention was implemented and data were collected over six months between September 2016 and February 2017. Sessions were offered weekly for the first five sessions and then bimonthly. Participants received an interactive, educational session, followed by a group-based motivational interviewing session to set and monitor dietary and physical activity goals. Participants were prescribed individualized calorie and fat goals through the mobile application, and 150 minutes of physical activity per week to achieve $10 \%$ weight loss.

\subsection{Phase 2 intervention adaptation rationale}

One limitation identified in the first phase of our study, conducted between September 2016 and February 2017, was the sample size of participants who committed to the program from baseline to post intervention. Although 42 African American participants were successfully enrolled in the program by church leaders, of the $16(38 \%)$ that completed baseline data collection, ${ }^{[14]}$ only three participants completed the program and post data collection. Therefore, phase 2 of the Church Bridge Project was informed by the phase 1 intervention results, which was adapted to improve participant retention and engagement of younger adult, African Americans in a weight management intervention. The methods, including rationale for program adjustment/adaptation based on phase 1 outcomes, and results for phase 2 are reported in this manuscript.

\subsection{Phase 2 sample}

The target community was similar to the phase 1 target community with a population of 32,647 reported in 2015. Of which, $36.5 \%$ are black and approximately $54.1 \%$ of the population is between the ages of 18 and 54 years with a median age of 31.9 years and a median income of $\$ 36,416 .{ }^{[15]}$ The 
study targeted young to middle (18 to 50 years) aged adults. Exclusion criteria included non-adults, those who did not identify as African American, individuals with a body mass index $<30 \mathrm{~kg} / \mathrm{m}^{2}$, and those who could not provide a signed medical clearance for participation.

\subsection{Phase 2 recruitment}

Church members were most successful at recruiting participants for the first phase of this church-based program. During Phase 2 intervention planning, community partners reported that they did not foresee being able to recruit more participants for the program from their county due to numerous reasons, including saturation of church leader recruiters and reported participant conflicts of having busy schedules, lack of commitment, and lack of interest. Thus, the executive team, including church leaders, made the decision to relocate the health program to a neighboring county with similar demographics. The community co-investigator identified a fourth church to serve as the host church for Phase 2.

Similar to Phase 1, church recruitment strategies included church leaders' word-of-mouth and nutrition education recruitment events delivered by student research scholars. The recruitment events occurred before Bible Study events and the student team set up a booth with study recruitment and nutrition education materials and sample healthy snacks and beverages. Church members and students collected contact information from interested potential participants, followed up with a phone call to initiate the registration process and request that eligible participants complete a web-based orientation and consent process. One participant self-registered for the program online using a tablet provided at one of the recruitment events. Due to time restraints, all recruitment activities were limited to one month duration prior to intervention implementation.

Outpatient primary care offices (O-PCP) proved to be difficult to engage for patient recruitment with $0 \%$ of participants referred from clinics. In an attempt to enhance engagement of O-PCP clinics, researchers partnered with a medical school at a local university and residency program at a local hospital/clinic that served the target population for phase 2 of our program. Referral cards were provided to the clinic for physicians to identify and refer eligible patients with the chief resident assigned to coordinate recruitment activities among approximately 18 other residents.

\subsection{Phase 2 program adjustment}

The phase 1 intervention was originally designed to be 12 sessions delivered over the course of six months, to alleviate the church of intense (weekly) program delivery. ${ }^{[13]}$ During phase 1, we identified program completion and re- tention as one of the biggest challenges. Since we saw the greatest decrease after the phase 1 delivery transitioned from weekly to bimonthly delivery, phase 2 intervention sessions were delivered over a shorter time period to result in total intervention delivery over eight weeks versus six months for Phase 1. Trained students delivered the intervention and conducted data collection and participant management activities. Participants in phase 1 indicated that "make-up sessions" would be beneficial in the future; therefore, sessions were live-streamed with recordings available for a limited time period through the Bridge $2 \mathrm{U}$ mobile application. Last, the original orientation process was a face-to-face session with paper-based consent forms; we streamlined this process with a two-step, web-based process to include the first step of screening participants and the second step to include orientation and consent. The web-based screening process, completed by participants or research staff, collected study eligibility and contact information. The orientation and consent process included an orientation video of the principal investigator explaining the project and consent process, as well as, an accessible copy of the medical clearance form and an electronic consent agreement. Eligible participants were e-mailed or texted (based on personal preference) a link to access orientation and consent materials.

\subsection{Measures}

Demographics, weight, height, and medical and weight histories were collected at baseline. Instruments deemed valid and reliable during previous research were used at pre- and post-intervention periods to measure self-efficacy ${ }^{[13]}$ and social support ${ }^{[17]}$ for diet and physical activity, physical activity levels, ${ }^{[18]}$ health-related quality of life,${ }^{[19]}$ and 24-hour recall data. Weight and girth circumferences (abdomen, waist, and hip measured below the last rib, above the iliac crest and at the widest point of the buttocks, respectively) were collected pre- and post-intervention and at each session attended. All data were collected by trained research staff through the Bridge2U web and mobile application system. ${ }^{[13]}$ Mean scores were calculated for obesity-related disease and family disease risk ("yes" or "no" responses to diagnosis with hypertension, diabetes, reflux, heart attack/stroke, high cholesterol and high triglycerides), and weight-related behaviors (checking weight with a scale, eating breakfast, exercising, and recording food intake). A "yes" response was scores as "1" and a "no" response was scored as " 0 " and a mean was calculated across items.

\subsection{Statistical analysis}

For phase 1, only three of 16 (18.8\%) participants completed both the pre- and post-intervention surveys. Descriptive statistics were used to analyze individual participant session attendance, anthropometric mean and total changes, and 
demographic data regarding participants who continued to participate into the second half of the program (sessions 6-12) versus participants who dropped out of the program within the first 5 sessions. Additionally, baseline data for age, education, income, gender, social support, self-efficacy, weightrelated behaviors, disease and family disease risk were examined. Fisher's exact and Wilcoxon signed-rank tests were used to examine baseline differences between participants who did and did not continue to participate with significance considered at $\alpha \leq 0.05$. For phase 2, baseline descriptive statistics were compared to data representing the general target population. Wilcoxon signed-rank tests determined post-intervention differences from baseline among continuous outcome variables (anthropometrics, diet and physical activity) whereas Cochran-Mantel-Haenszel test was used to examine pre- and post-changes in stage of change.

Table 1. Phase 1 comparison of anthropometrics, age, disease diagnosis, family disease risk, weight behaviors and demographics between participants who dropped out and those retained in the study

\begin{tabular}{|c|c|c|}
\hline & $\begin{array}{l}\text { Participants who dropped } \\
\text { out within first } 5 \text { sessions } \\
(n=11)\end{array}$ & $\begin{array}{l}\text { Participants who participated in } \\
\text { the } 2^{\text {nd }} \text { half of the program } \\
(n=5)\end{array}$ \\
\hline Continuous Variables & \multicolumn{2}{|c|}{ Mean (standard deviation) } \\
\hline Age (years) & $36.36(7.76)$ & $34.80(9.60)$ \\
\hline Body Mass Index $\left(\mathrm{kg} / \mathrm{m}^{2}\right)^{\mathrm{a}}$ & $37.21(5.43)$ & $43.24(5.11)$ \\
\hline Weight (kg) & $99.25(17.64)$ & $116.81(19.96)$ \\
\hline Abdomen $(\mathrm{cm})$ & $110.69(15.65)$ & $124.21(12.12)$ \\
\hline Waist (cm) & $99.44(11.23)$ & $106.68(7.67)$ \\
\hline $\operatorname{Hip}(\mathrm{cm})^{\mathrm{a}}$ & $119.76(13.36)$ & $135.13(13.34)$ \\
\hline Disease Diagnosis (mean score) ${ }^{\mathrm{b}}$ & $0.14(0.15)$ & $0.23(0.09)$ \\
\hline Family Disease Risk (mean score) $^{\mathrm{b}}$ & $0.35(0.31)$ & $0.25(0.28)$ \\
\hline Weight Behaviors (mean score) ${ }^{\mathrm{b}}$ & $0.18(0.34)$ & $0.35(0.22)$ \\
\hline Categorical Variables & \multicolumn{2}{|c|}{$\mathrm{n}(\%)$} \\
\hline Female & $8(61.5)$ & $5(100.0)$ \\
\hline Completed high school and/or some college but no degree & $2(18.2)$ & $3(60.0)$ \\
\hline Individual annual income less than $\$ 40,000.00$ & $6(54.5)$ & $3(60.0)$ \\
\hline Married & $5(45.5)$ & $1(20.0)$ \\
\hline
\end{tabular}

Note. a. Group mean differences are significantly different, $\mathrm{P} \leq .05$, b. Overall baseline $(\mathrm{n}=16)$ Disease Diagnosis, Family Disease Risk and Weight Behaviors scores were $0.17(0.14), 0.68(0.29)$ and $0.23(0.32)$, respectively.

\section{RESUlts}

\subsection{Phase 1 post-intervention results}

The mean attendance rate for all 12 intervention sessions was $39.9 \%$ (61.4\% for the first five sessions, followed by $24.5 \%$ for the remaining sessions). The mean attendance rate for each session per participant was $15.8 \%$ (31.6\% for the first five sessions, followed by $15.9 \%$ for the remaining sessions). Mean BMI $\left(\mathrm{kg} / \mathrm{m}^{2}\right)$, weight $(\mathrm{kg})$, and waist, abdomen and hip circumferences (centimeters, $\mathrm{cm}$ ) showed decreases at the 5 th session and post-intervention (see Table 1). Mean weight lost for participants at the 5 th and post-intervention session accounted for 0.46 and $5.6 \%$, respectively, of baseline body weight. Those who dropped had significantly lower mean BMI (42.06 versus $45.3 \mathrm{~kg} / \mathrm{m}^{2}, p=.047$ ) and hip circumference (132.59 cm versus $140.12 \mathrm{~cm}, p=.048$ ) compared to those who did not. Those who persisted in the program beyond the 5th session were slightly younger and had lower family disease risk scores, and higher disease and weight- related behavior scores (see Table 1); they were also female, similar in education and income status to those who dropped within the first five sessions, and more likely to report a marital status of "Single" (see Table 1).

Table 2. Examination of Phase 2 study population generalizability to the broader target population of interest

\begin{tabular}{llll}
\hline & $\begin{array}{l}\text { Phase 2 } \\
\text { Participants }\end{array}$ & $\begin{array}{l}\text { Target } \\
\mathbf{2 0 1 6}^{[32]}\end{array}$ & Population, \\
\hline African American (\%) & 100.0 & 36.7 \\
Female (\%)a & 52.4 & 52.2 \\
Uninsured (\%)a & 14.3 & 27 \\
Median age (years) a & 42.0 & 32.0 \\
At least some college (\%) & 57.1 & 42.9 \\
Obesity prevalence (\%) & 100.0 & 34.0 \\
Diabetes prevalence (\%) & 0.0 & 11.0 \\
Poor to fair health (\%) & 35.7 & 11.1 \\
Smokers (\%) & 7.1 & 22.0 \\
\hline
\end{tabular}

Note. ${ }^{\mathrm{a}} \mathrm{n}=21$, data represents enrollment sample; remainder of data represents participants $(n=14)$ who completed baseline data collection. 
Table 3. Phase 2 pre- and post-intervention means for anthropometrics, stage of change, diet and physical activity behaviors $(\mathrm{n}=11)$

\begin{tabular}{llll}
\hline Variable & $\begin{array}{l}\text { Pre-Intervention Mean } \\
\text { (standard deviation) }\end{array}$ & $\begin{array}{l}\text { Post- Intervention } \\
\text { Mean (standard deviation) }\end{array}$ & $\boldsymbol{p}$ \\
\hline Anthropometrics & $17.04(1.64)$ & $15.83(1.78)$ & $<.01$ \\
$\quad$ Abdomen (centimeters, cm.) & $18.62(1.79)$ & $17.84(1.87)$ & $<.01$ \\
Hip (cm.) & $15.85(1.31)$ & $14.51(1.80)$ & $<.01$ \\
Waist (cm.) & $98.09(16.43)$ & $93.34(15.83)$ & .03 \\
$\quad$ Weight (kilograms) & $35.07(6.58)$ & $33.90(6.63)$ & .02 \\
$\quad$ Body Mass Index $\left(\mathrm{kg} / \mathrm{m}^{2}\right)$ & $3.67(1.15)$ & $4.09(.30)$ & .03 \\
Stage of Change & & & .05 \\
Physical Activity (IPAQ) & $236.67(204.46)$ & $440.00(392.82)$ & .19 \\
$\quad$ Moderate & $611.43(737.63)$ & $1,003.64(1782.53)$ & .04 \\
$\quad$ Vigorous & $298.26(406.31)$ & $668.91(934.75)$ & .04 \\
$\quad$ Walk & $1,215.04(1011.42)$ & $2,011.73(2324.04)$ & \\
$\quad$ Total & $1,192.96(221.89)$ & $1,108.86(221.99)$ & 0.89 \\
Reported Diet Intake & $39.9(14.8)$ & $40.5(16.26)$ & 0.14 \\
$\quad$ Total Energy (kilocalories) & & & \\
$\quad$ Fat (grams) & & & \\
\hline
\end{tabular}

\subsection{Phase 2 intervention outcomes}

A total of 26 participants were reached by two methods, referrals from church leaders and recruitment from student scholar outreach activities. Fifteen individuals were referred to research staff from church leaders. Of the 15,11 individuals completed the screening process. Student scholars recruited eight participants, and six (out of eight) participants were screened by research staff and one participant completed the self-screening process using the web link. Three additional individuals were referred by enrolled participants and of the three, two completed screening. Of the 26 participants reached, 21 (80.8\%) completed the screening process. Of approximately 100 eligible patients seen over the one month recruitment period, zero participants were recruited from the health clinic. Proportions of male and female participants enrolled were equal (52.4\% female versus $47.6 \%$ male) and most participants reported having a family physician $(76.2 \%)$ and insurance $(85.7 \%)$ and received a wellness visit in the past year (71.4\%). Of the 21 screened participants, 14 individuals (66.7\%) completed the orientation and consent process and baseline survey data collection and 12 of the 14 participants (57.1\%) completed baseline anthropometric data. Baseline demographics and participant generalizability are reported in Table 2. Baseline mean ( \pm standard deviation) scores for obesity-related disease risk, family disease risk, and weight-related behaviors were 0.29 $(0.14), 0.59(0.25)$ and $0.28(0.26)$, respectively. Nonparametric (Wilcoxon sign-ranked and Fisher's exact) tests showed no differences in screening variables between enrolled $(\mathrm{n}=$ 14) and unenrolled $(\mathrm{n}=7)$ participants (data not shown). 10 and 11 participants completed post-intervention survey and

Published by Sciedu Press anthropometric data collection, respectively, for a retention rate of $78.6 \%$. Since only one person did not return after the 5th session and sessions were weekly, reporting data for the first and second half of the program was not useful to examine as it was for phase 1 to inform intervention adaptation and thus, the information is not reported.

The mean attendance rate for all 12 intervention sessions was $55.4 \%$ and the attendance rate for each session per participant was $51.1 \%$. Statistically significant reductions were noted among girth circumferences, weight and BMI postintervention; a mean $4.8 \%$ reduction of baseline body weight was observed. Statistically significant increases were also noted among stage of change for weight loss and total physical activity variables. There were no significant changes in total energy and dietary fat grams. See Table 3 for detailed results.

\section{Discussion}

The adaptations to a pilot intervention and results were compared between two phases of a weight management intervention targeting young to middle aged African Americans in Mississippi. Results showed significant pre-post improvements in weight and girth circumferences for both phases of intervention; phase 2 results also supported higher stages of change for weight loss and increased physical activity levels. Phase 1 participants who continued into the second half of the program had a higher BMI and reported a higher obesity-related disease prevalence and greater engagement in weight-related behaviors. Participants of the second half of the phase 1 program were single females with similar 
education and income levels to those that dropped out. Attendance of intervention sessions and attrition were improved in the second phase of intervention with no noticeable attrition pattern after the 5th session.

While improvements in participant retention and engagement were observed from phase 1 to phase 2 , it should be noted that neither study achieved the goal $n=20$ which underscores the recruitment difficulty of the target population, even in the state with the highest obesity prevalence across the nation. Other church-based studies ${ }^{[20-23]}$ with an all or predominantly African American study sample had higher retention (range of 73.0 to $84.0 \%$ ) and older participants (mean age range of 47 to 60 years of age) compared to our phase 1 study while retention was comparable for phase 2 . Additionally, the research reflects that most participants in similar studies were predominantly female and overweight/obese. Participants of three of the five ${ }^{[20-24]}$ similar studies were at risk for diabetes $^{[22]}$ or were previously diagnosed with hypertension ${ }^{[23]}$ or diabetes ${ }^{[24]}$ as inclusion criteria. Overall, participants of similar studies ${ }^{[20-24]}$ were older and had a greater prevalence of chronic disease compared to our baseline samples. ${ }^{[13]}$

\subsection{Lesson Learned}

From observation and comparison of phase 1 and 2 intervention findings among this population, we have identified four key lessons learned from these results to consider for future research:

(1) Disease diagnosis may be a key motivating factor for obesity management participation among young to middle aged adult African Americans. In the phase 1 sample, those who continued into the second half of the program had higher BMI and prevalence of disease (albeit the latter was not significant) compared to those who dropped out in the first half. Since other study samples had older populations with greater disease prevalence, a chronic disease diagnosis may influence motivation for completing weight management programs. Our phase 2 intervention did have improved retention (comparable to similar literature) and those participants also had a higher prevalence of disease compared to phase 1 participants but family disease risk was lower and participation in weight-related behaviors was similar.

(2) More intensive interventions may improve participant retention and outcomes. The program structures reported by similar studies differ. For example, most offer at least eight weekly sessions before extending the session frequency to bimonthly. Thus, our phase 1 program format may have prematurely extended sessions to bimonthly intervals. The phase 2 intervention offered weekly sessions, which were also live-streamed with recordings accessible for a limited time, and exhibited a much more successful retention rate. While a greater weight decrease was noted in phase 1 , the difference likely produces no clinical significance. Additionally, phase 2 outcomes were observed over a shorter time period and thus, it could be interpreted that phase 2 may have produced greater results since it achieved nearly the same weight outcome as phase 1 in about half the time. Recent literature reports that as little as $5 \%$ of weight loss is associated with improvements in blood pressure and cholesterol profiles as well as decreased health care costs. ${ }^{[25]}$ While studies representing African American adults enrolled in behavioral interventions is very limited, ${ }^{[26]}$ reviews have found intensive obesity management programs to produce clinically meaningful weight loss. ${ }^{[27,28]}$

(3) Technology facilitated interventions may improve participant engagement and ultimately, retention. Our study observed a $15.5 \%$ improvement in phase 2 intervention attendance versus phase 1 as well as a 59.8\% improvement in retention. While we cannot determine whether this was a result of a change in program format or introduction of live-streamed sessions, we hypothesize the technology was a major factor. The theory was only anecdotally supported by focus group data, currently being prepared for publication, and requires further investigation to establish the role that technology may play in supporting and enhancing weight management interventions.

(4) Churches were key stakeholders to reach underserved, young to middle aged African Americans. In both phases, zero participants were recruited from primary outpatient health care centers and all were recruited from communitybased recruitment methods. This is a critical consideration for research and practice. Churches continue to be most successful for recruitment of African Americans, ${ }^{[8]}$ and even among young to middle aged adults who are underrepresented in the church-based and rural, minority health literature ${ }^{[10,11]}$ Considering minority health care practice, policies that place emphasis on obesity management in provision to a primary care setting may not reach and alleviate disparities among African Americans residing in Deep South states. Mississippi (for example) is one of the most underserviced states in the country with the majority of the state considered a medically underserviced and health care professional shortage - primary care physician area. ${ }^{[2,30]}$

\subsection{Limitations}

Although observations from phase 1 of the study were informative, sample size was inadequate for inferential analysis, which makes it difficult to draw any firm conclusions or relationships. Yet, the phase 1 intervention did inform adaptations to the phase 2 intervention where improvements 
were noted. Additionally, variability in phase 1 session attendance made it difficult to ascertain intervention effects on individuals who dropped out before the 5th month and whether dropout was related to lack of weight loss success or insufficient program commitment. While the intervention effectiveness results showed promise, the tool used to collect dietary data was new and may have impacted the data that was collected; we also could not account for a potential learning effect related to using the tool over time. There were also two key changes to the phase 2 intervention (live-streamed sessions and program format changes) that made it difficult to determine the added effect of each strategy. In other words, it cannot be determined whether a live-streamed version of the bi-monthly format would be as effective at program engagement as the live-streamed, weekly format. Additionally, it should be noted that neither intervention phase achieved the goal $n=20$, which may have been possible for phase 2 if given a longer recruitment period than one month. Last, the reported dietary data showed no changes pre-post intervention, which is unlikely since weight loss was objectively presented. Our previous research ${ }^{[31]}$ shows that the mobile recall method used results in significant underreporting which combined with the learning effect of logging dietary data over time may have resulted in unreliable data.

\section{Conclusion}

Our findings are important for advancing research and practice initiatives targeting obesity reduction and management among African Americans. Minimal information is available regarding approaches toward implementing church-based, disease prevention interventions among younger African American adults. This study examined issues related to and provides important considerations for engaging younger adult African Americans in a weight-management program. Results inform future research, including weight management intervention protocol and retention strategies as well as how technology may improve program retention and participation.

\section{FUNDING}

Research reported in this publication was supported by the National Institute On Minority Health And Health Disparities of the National Institutes of Health under Award Number 15MD010213. The content is solely the responsibility of the authors and does not necessarily represent the official views of the National Institutes of Health.

\section{ACKNOWLEDGEMENTS}

We would like to acknowledge the church pastors and leaders who were integral to the development, planning and recruitment for the program. We extend our appreciation to all the graduate and undergraduate students who contributed to program delivery and data collection.

\section{CONFLicts OF InTEREST Disclosure}

The authors declare no conflict of interest.

\section{REFERENCES}

[1] Mozaffarian D, Benjamin EJ, Go A, et al. Stroke Statistics Subcommittee. (2016). Executive Summary: Heart Disease and Stroke Statistics-2016 Update: A Report From the American Heart Association. Circulation. 2016; 133(4), 447-54. PMid:26811276. https: //doi.org/10.1161/CIR.0000000000000366

[2] Centers for Disease Control and Prevention. Overweight and Obesity. Retrieved June 26, 2017. Available from https://www.cdc.gov/ obesity

[3] Nutrition and Weight Status Healthy People 2020. (n.d.). Retrieved June 26, 2017. Available from https: //www.healthypeople.gov/2020/topics-objectives/to $\mathrm{pic/nutrition-and-weight-status/objectives \# 4968}$

[4] LaVeist T, Pollack K, Thorpe R, et al. Place, not race: disparities dissipate in southwest Baltimore when blacks and whites live under similar conditions. Health affairs (Project Hope). 2010; 30(10): 18807. PMid:21976330. https://doi.org/10.1377/hlthaff . 2011 .0640

[5] Thorpe RJ, Kelley E, Bowie JV, et al. Explaining Racial Disparities in Obesity Among Men. American Journal of Men's Health. 2015; 9(6): 464-472. PMid:25249452. https ://doi .org/10.1177/15 57988314551197

Published by Sciedu Press
[6] Trivedi T, Liu J, Probst J, et al. Obesity and obesity-related behaviors among rural and urban adults in the USA. Rural and remote health. 15(4), 3267. Retrieved from http://www.ncbi.nlm.nih.gov/p ubmed/26458564

[7] Johnson ER, Carson TL, Affuso O, et al. Relationship between social support and body mass index among overweight and obese African American women in the rural deep South, 2011-2013. Preventing chronic disease. 2014; 11, E224. PMid:25539128. https : //doi.org/10.1007/s11906-017-0777-3

[8] Lemacks J, Wells BA, Ilich JZ. Interventions for improving nutrition and physical activity behaviors in adult African American populations: a systematic review. 2013. PMid:23786910. https: //doi.org/10.5888/pcd10.120256

[9] Lancaster KJ, Carter-Edwards L, Grilo S, et al. Obesity interventions in African American faith-based organizations: a systematic review. Obesity Reviews. 2014; 15: 159-176. PMid:25196412. https://doi.org/10.1111/obr.12207

[10] Cené CW, Haymore LB, Ellis D, et al. Implementation of the Power to Prevent Diabetes Prevention Educational Curriculum Into Rural African American Communities. The Diabetes Educator. 2013; 39(6): 776-785. PMid:24129595. https://doi.org/10.1177/014572 1713507114 
[11] Mayer-Davis EJ, D’Antonio AM, Smith SM, et al. Pounds off with empowerment (POWER): a clinical trial of weight management strategies for black and white adults with diabetes who live in medically underserved rural communities. American journal of public health. 2004; 94(10): 1736-42. https://doi.org/10.2105/AJ PH. 94.10.1736

[12] Perri MG, Limacher MC, Durning PE, et al. Extended-care programs for weight management in rural communities: the treatment of obesity in underserved rural settings (TOURS) randomized trial. Archives of Internal Medicine. 2008; 168(21): 2347-2354. PMid:19029500. https://doi.org/10.1001/archinte.168.2 1.2347

[13] Lemacks JL, James RE, Abbott L, et al. The Church Bridge Project: An Academic-Community Perspective of a Church-Based Weight Management Pilot Intervention among Young Adult African Americans. Progress in Community Health Partnerships: Research, Education and Action. 2008; 12(1S): 23-34. PMid:29755046. https: //doi.org/10.1353/cpr.2018.0018

[14] Lemacks J, Landry A, Wenzler P. Formative Research to Identify Community Partnerships and Foster Relationships for Health Promotion Research in South Mississippi. Public Health. 2017; 159: 58-62. PMid:29609837. https ://doi.org/10.1016/j.puhe.2018.01 .023

[15] Forrest County, MS I Data USA. (n.d.). Retrieved December 3, 2018. Available from https://datausa.io/profile/geo/for rest-county-ms/\#top

[16] Sallis JF, Pinski RB, Grossman RM, et al. The development of self efficacy scales for health-related diet and exercise behaviors. Health Education Research. 1988; 3(3): 283-292. https://doi.org/10 .1093/her/3.3.283

[17] Sallis JF, Grossman RM, Pinski RB, et al. The development of scales to measure social support for diet and exercise behaviors. Preventive Medicine. 1987; 16(6): 825-836. https ://doi.org/10.1016/00 91-7435 (87) 90022-3

[18] Booth ML, Ainsworth BE, Pratt M, et al. International physical activity questionnaire: 12-country reliability and validity. Medicine \& Science in Sports \& Exercise. 2003; 195(9131/03): 1381-3508. PMid:12900694. https://doi.org/10.1249/01.MSS.0000078 924.61453. FB

[19] Stewart AL, Hays RD, Ware JE. The MOS short-form general health survey. Reliability and validity in a patient population. Medical care. 1988; 26(7): 724-35. PMid:3393032. https://doi .org/10.109 7/00005650-198807000-00007

[20] Tussing-Humphreys, LM, Thomson JL, Onufrak SJ. A ChurchBased Pilot Study Designed to Improve Dietary Quality for Rural, Lower Mississippi Delta, African American Adults. Journal of Religion and Health. 2015; 54(2): 455-469. PMid:24442772. https://doi.org/10.1007/s10943-014-9823-5

[21] Thomson JL, Goodman MH, Tussing-Humphreys L. Diet Quality and Physical Activity Outcome Improvements Resulting From a Church-Based Diet and Supervised Physical Activity Intervention for Rural, Southern, African American Adults: Delta Body and Soul III. Health Promotion Practice. 2015. PMid:25603798. https://doi .org/10.1177/1524839914566851

[22] Tang TS, Nwankwo R, Whiten Y, et al. Outcomes of a Churchbased Diabetes Prevention Program Delivered by Peers: A Feasibility Study. The Diabetes Educator. 2014. PMid:24481174. https : //doi.org/10.1177/0145721713520569

[23] Skolarus LE, Cowdery J, Dome M, et al. Reach Out Churches: A Community-Based Participatory Research Pilot Trial to Assess the Feasibility of a Mobile Health Technology Intervention to Reduce Blood Pressure Among African Americans. Health Promotion Practice. 2017. PMid:28583024. https : //doi .org/10.1177/152483 9917710893

[24] Parra-Medina D, D'Antonio A, Smith SM, et al. Successful recruitment and retention strategies for a randomized weight management trial for people with diabetes living in rural, medically underserved counties of South Carolina: The POWER study. Journal of the American Dietetic Association. 2004. PMid:14702587. https://doi.org/10.1016/j.jada.2003.10.014

[25] Ryan DH, Yockey SR. Weight Loss and Improvement in Comorbidity: Differences at 5\%,10\%,15\%, and Over. Current obesity reports. 2017; 6(2): 187-194. PMid:28455679. https ://doi.org/10.100 7/s13679-017-0262-y

[26] Newton RL, Griffith DM, Kearney WB, et al. A systematic review of weight loss, physical activity and dietary interventions involving African American men. Obesity Review. 2014; 15, 93-106. PMid:25196408. https ://doi.org/10.1111/obr.12209

[27] Wadden TA, Butryn ML, Hong P, et al. Behavioral Treatment of Obesity in Patients Encountered in Primary Care Settings. JAMA 2014; 312(17): 1779. PMid:25369490. https://doi.org/10.1 $001 /$ jama. 2014.14173

[28] Wadden TA, Wolfe BM, Yanovski SZ, et al. AHA/ACC/TOS Guideline for the Management of Overweight and Obesity in Adults: A. 2013. Retrieved from http: //circ.ahajournals.org/content/early/2013/11/1 1/01.cir.0000437739.71477.ee.citation

[29] Health Resources \& Services Administration (HRSA). Medically Underserved Areas/Populations. Retrieved July 9, 2019. Available from https://data.hrsa.gov/ExportedMaps/MUA/HGD WMapGallery_MUA.pdf

[30] Health Resources \& Services Administration (HRSA). Health Professional Shortage Area (HPSA) - Primary Care Physician. Retrieved July 9, 2019. Available from https://data.hrsa.gov/Export edMaps/HPSAs/HGDWMapGallery_BHPR_HPSAs_PC.pdf

[31] Lemacks JL, Adams K, Lovetere A. Dietary Intake Reporting Accuracy of the Bridge2U Mobile Application Food Log Compared to Control Meal and Dietary Recall Methods. Nutrients. 2019; 11(1). PMid:30669430. https://doi.org/10.3390/nu11010199 